Aramendi Jauregui, P.; Arburua Goinetxe, R.M. y Buján Vidales, K. (2018). El aprendizaje basado en la indagación en la enseñanza secundaria. Revista de Investigación Educativa, 36(1), 109-124.

DOI: http://dx.doi.org/10.6018/rie.36.1.278991

\title{
El aprendizaje basado en la indagación en la enseñanza secundaria
}

\section{Investigation-Based Learning in Secondary Education}

\author{
Pello Aramendi Jauregui, Rosa María Arburua Goienetxe y Karmele Buján Vidales \\ Dpto. de Didáctica y Organización Escolar \\ Universidad del País Vasco
}

\begin{abstract}
Resumen
Las estrategias activas y contextualizadas de aprendizaje ofrecen la posibilidad de acercar el conocimiento aprendido a la vida real. El estudio que se presenta se ha realizado en los institutos de secundaria de la zona de San Sebastián (España) y la ciudad de Nantes (Francia). Su objetivo fundamental es describir las opiniones de los estudiantes de secundaria de ambas ciudades sobre aspectos genéricos del aprendizaje basado en la indagación. Los resultados indican que el alumnado consultado en el estudio, subraya la importancia de las estrategias de aprendizaje basadas en la indagación, la funcionalidad del aprendizaje, la búsqueda de información y el fomento de los aspectos afectivos y emocionales. El alumnado que ha superado todas las asignaturas confía en la labor educativa del centro escolar, le gusta leer, escribir, participar en clase y autoevaluar su propio trabajo. Caminar hacia una educación de calidad, donde todo el alumnado desarrolla al máximo sus posibilidades, exige replantear el papel del docente y del estudiante en el fomento de la indagación y en el desarrollo de competencias emocionales en los procesos de enseñanza y aprendizaje como dos de los pilares básicos de la educación del futuro.

Palabras clave: aprendizaje basado en la indagación; currículum; educación secundaria; competencias emocionales.
\end{abstract}

Correspondencia: Rosa María Arburua Goienetxe, rosamaria.arburua@ehu.eus, Universidad del País Vasco, Facultad de Educación, Filosofía y Antropología. Avenida de Tolosa 70, 20018. 


\begin{abstract}
Active and contextualized learning strategies provide the possibility of bringing the knowledge learned closer to real life. The study presented here has been carried out in secondary schools in the San Sebastian area (Spain) and the city of Nantes (France). Its fundamental aim is to describe the opinions of secondary school students in both cities regarding generic aspects of research-based learning. Results show that students consulted in the study highlight the importance of investigation-based learning strategies and those based on the functionality of learning, the search for information and the promotion of affective and emotional factors of learning. Students who have passed in all their subjects have confidence in the educational work of the school, they like reading and writing, participating in class and self-evaluating their work. Moving towards quality education, where all students develop their possibilities to the maximum, requires a new approach to the roles of teacher and student based on the promotion of research and the development of emotional skills in teaching and learning processes as two of the basic building blocks of the education of the future.
\end{abstract}

Keywords: curriculum; emotional skills; investigation-based learning; secondary education.

\title{
Introducción
}

La motivación de los estudiantes por el aprendizaje es un elemento clave si se pretende que la ciudadanía aprenda a lo largo de la vida. El acercamiento de los procesos de enseñanza y aprendizaje a la vida real y la percepción por parte de los estudiantes de la utilidad de lo aprendido son dos elementos importantes que impulsan el interés por el aprendizaje. Andriessen, Phalet y Lens (2006) analizan la influencia de la utilidad del conocimiento aprendido en la mejora del rendimiento de los estudiantes de grupos desfavorecidos y no desfavorecidos. Los resultados indican que comprender la utilidad de lo que se aprende aumenta la motivación en los estudios por parte de los dos colectivos. En la misma línea, Lavonen y Laaksonen (2009) constatan que el rendimiento académico de los estudiantes finlandeses mejora cuando valoran la utilidad de la asignatura para desempeñar futuras profesiones. Según los autores, los elementos fundamentales de la metodología docente son las actividades prácticas, las demostraciones y la posibilidad de que los estudiantes puedan extraer conclusiones durante y después de finalizar el proceso de aprendizaje.

La situación de la educación secundaria española es bien diferente. Mengascini y Mordeglia (2014) opinan que las actividades prácticas se llevan a cabo con poca frecuencia en los centros educativos de primaria y secundaria. El trabajo experimental es escaso, las actividades realizadas remiten a objetivos de conocimiento conceptual y las prácticas se basan fundamentalmente en la observación. Generalmente, el objetivo de las prácticas de clase es confirmar la solución de un problema que ha sido tratado en una lección magistral. A menudo, en estas prácticas, se aplica la receta correspondiente para llegar a una conclusión prefijada, sin clarificar para qué sirve y cuál es su utilidad en la vida real. Esta afirmación también es compartida por Doménech (2012) cuando analiza los enfoques de enseñanza del profesorado de primaria y secundaria 
españoles. Los estilos de pensamiento predominantes en el profesorado de la muestra son de tipo ejecutivo y jerárquico. Según el autor, los docentes consultados utilizan un enfoque de enseñanza centrado en el profesor y la profesora y en la transmisión del conocimiento. Lamentablemente, aún se deben superar muchos obstáculos para lograr que la enseñanza secundaria sea una etapa atractiva. En este sentido, Criado y Guzmán (2014) comparan el currículo de ciencias español de la educación obligatoria (enseñanzas mínimas nacionales) con el de Inglaterra y EEUU. Opinan que se está produciendo un declive en el interés por las ciencias debido a la lejanía entre los conocimientos impartidos y las propias experiencias de los estudiantes. Bermúdez, Díaz, Lía y Gavidia (2014) analizan el tratamiento de diversos temas y conceptos en los libros de texto de la educación secundaria. Según los autores, los materiales didácticos analizados en su estudio reflejan una multiplicidad de definiciones desfasadas, apreciándose igualmente confusiones terminológicas, lo que pone en evidencia su escasa actualización.

\section{La indagación: ¿una alternativa a la enseñanza centrada en el docente?}

Si se pretende mejorar los niveles de éxito de la educación secundaria, el aprendizaje debe convertirse en un proceso útil y motivador para el estudiante. Vílchez y Bravo (2015) afirman que, en función del rol del docente y del estudiante, los procesos de indagación en el aula pueden ser de tres tipos: la indagación estructurada (el docente plantea tanto el problema como el procedimiento a seguir), la indagación guiada (el docente plantea el problema y el alumnado decide cómo resolverlo) y la indagación abierta (tanto el problema como el método de resolución parten de los estudiantes). Los autores concluyen que los docentes de educación secundaria utilizan mayoritariamente actividades de indagación estructurada en su práctica habitual. Se debería formar a los docentes para desarrollar estrategias de enseñanza y aprendizaje basadas en la indagación guiada y abierta y, de esta manera, mejorar la motivación y la implicación de los estudiantes.

En el aprendizaje basado en problemas (Problem Based Learning-PBL en adelante), el rol del docente y del estudiante también cambia de forma sensible. Es una estrategia que intenta otorgar más importancia al rol del estudiante como principal responsable de su propio proceso de aprendizaje. Recio (2014) constata que, con la metodología PBL, los estudiantes se convierten en investigadores y el docente en un entrenador, que ayuda a los grupos de clase mediante preguntas, orienta la búsqueda de información, fomenta el análisis y la síntesis, conoce la materia y su conexión con el mundo real y guía el aprendizaje hacia la indagación y la resolución de problemas.

Prieto et al. (2006), sensibilizados con el impulso de la indagación en las aulas, desarrollan una versión adaptada del aprendizaje basado en problemas (denominada PBL 4X4). Ésta consta de cuatro fases (análisis, investigación, resolución de problemas y evaluación) y cuatro escenarios de trabajo (individual, grupal sin tutor, grupal con tutor y clase completa). La evaluación demostró que las actividades desarrolladas fueron motivadoras para el alumnado y que su carga de trabajo aumentó, aproximadamente, en un ochenta por ciento. Chen y Howard (2010), Gillies (2008), Lara 
y Samper (2015), Studsrod y Bru (2011) opinan que la metodología PBL, pretende que el alumnado pase de memorizar datos a producir un discurso argumentativo con el cual valida las conjeturas planteadas. Los resultados indican que la actitud ante el aprendizaje y el clima de aula mejoran de forma considerable.

Por otra parte, Dignath y Buettner (2008) y Zamora y Ardura (2014) constatan que el esfuerzo que debe realizar el profesorado para comprender las razones implícitas de las ideas que expresa el alumnado y de sus inconsistencias, facilita la detección de sus necesidades y concreta su actuación docente. Trabajar los procesos metacognitivos conjuntamente con los estudiantes mejora la implicación de éstos con los procesos de aprendizaje en el área de matemáticas (Gasco, 2016). A nivel afectivo y emocional, el alumnado interpreta estas estrategias metacognitivas como un acto de compromiso del profesorado con su formación (Hinojosa \& Sanmartí, 2015). Los aspectos emocionales, por consiguiente, también están vinculados con el aprendizaje basado en la indagación. Di Giusto, Martin y Arnaiz (2014); Martínez, Piqueras e Inglés (2011); Reschly, Huebner, Appleton y Antaramian (2008) y Sideridis y Kaplan (2011) analizan la relación de los aspectos emocionales con la capacidad de afrontar situaciones complejas. Los estudios muestran que niveles altos de inteligencia emocional se relacionan con estrategias de afrontamiento basadas en la reflexión y la resolución, mientras que niveles bajos se vinculan a estrategias basadas en la evitación, la queja y la superstición.

Por otro lado, se debe remarcar que la implantación en los centros educativos de estrategias indagadoras de enseñanza y aprendizaje no es una tarea sencilla. Las resistencias al cambio, el aumento de la carga de trabajo para obtener logros que no se perciben de forma inmediata, la ansiedad de los docentes (generalmente por falta de formación), las dificultades de aplicación en clases numerosas y la falta de planificación y trabajo en equipo condicionan la viabilidad de los procesos de innovación (Abel, 2012). El autor opina que la cultura curricular y organizativa del centro educativo condiciona la implantación de innovaciones en el currículum y, en este caso, de las estrategias de aprendizaje basadas en la indagación.

Precisamente la implantación progresiva de esta estrategia de enseñanza y aprendizaje en centros educativos de secundaria de dos regiones de países diferentes (Bretaña y País Vasco) fue el motivo del planteamiento de este estudio. La región de Bretaña (Breizh) se encuentra ubicada en el noroeste de Francia (departamento de Loire Atlantique) y tiene una población aproximada de 3 millones de habitantes. Las escuelas Diwan pertenecen a una red de centros educativos federados de esta región francesa, creada en la década de los setenta (1978, Nantes) y tienen como cometido fundamental la recuperación de la lengua y cultura bretonas. El gobierno francés les denegó el estatus público porque se dedicaban a enseñar el bretón "una lengua que va en contra de la ley" en "unos centros que reducen la enseñanza del francés" (La Vanguardia, 29 de noviembre 2002, p. 1). También han participado centros de ESO (Educación Secundaria Obligatoria) de la zona de San Sebastián que utilizan fundamentalmente el euskera en clase. Todos los centros educativos seleccionados han manifestado su intención de implantar progresivamente estrategias activas de 
aprendizaje. En este primer estudio, y ante las diferencias en el currículum entre ambas regiones, se ha optado por analizar las preferencias de los estudiantes en torno a las actividades de aprendizaje basadas en la indagación.

\section{Método}

\section{Objetivos}

El aprendizaje basado en la indagación es una metodología de trabajo adecuada para el desarrollo de competencias básicas en los estudiantes de la enseñanza obligatoria (Vilchez \& Bravo, 2015). El desencadenante de este estudio ha sido el interés mostrado por algunos centros educativos de Bretaña y el País Vasco por mejorar la enseñanza y desarrollar, a medio plazo, metodologías de trabajo más adaptadas a las preferencias de su alumnado y, concretamente, basadas en la indagación y abordaje de situaciones complejas.

En la investigación se plantearon los siguientes objetivos específicos:

- Analizar las características del alumnado de educación secundaria obligatoria participante en el estudio.

- Describir las opiniones de los estudiantes de las dos regiones (Bretaña y País Vasco) sobre las estrategias de enseñanza y aprendizaje basadas en la indagación.

- Analizar y comparar las puntuaciones obtenidas por los estudiantes con y sin problemas académicos en relación con las actividades de aprendizaje desarrolladas en el aula.

\section{Participantes}

La investigación se ha realizado en centros educativos de educación secundaria de la región de Bretaña (zona de Nantes) y el País Vasco (zona de San Sebastián). Los centros de ambas zonas acaban de iniciar una experiencia de innovación centrada en el aprendizaje basado en la indagación y comparten, además, similitudes en lo referente a las finalidades de su proyecto educativo (preservar y fomentar el idioma y la cultura autóctona). Se han enviado cuestionarios a 5 centros de secundaria con modelos educativos trilingües (bretón, francés e inglés) existentes en la zona de Nantes (Escuelas Diwan) y a 5 institutos de los alrededores de San Sebastián (euskera, castellano e inglés). Se pretende analizar, entre otras cuestiones, la predisposición inicial y la satisfacción de los estudiantes hacia estas estrategias indagadoras de aprendizaje. Los estudiantes pertenecen a centros educativos con estatus socioeconómicos similares.

En el estudio han participado estudiantes entre 12 y 15 años de edad. Contestaron al cuestionario 568 estudiantes de la zona de Nantes (Bretaña) y 452 estudiantes de la zona de San Sebastián (País Vasco). Han participado el 73.27\% de los estudiantes de los centros educativos seleccionados. 
Tabla 1

Muestra de la investigación

\begin{tabular}{cccc}
\hline Región & $\begin{array}{c}\text { Centros de } \\
\text { secundaria }\end{array}$ & $\begin{array}{c}\text { Participantes } \\
\text { Estudiantes }\end{array}$ & $\begin{array}{c}\text { N Total } \\
\text { Estudiantes }\end{array}$ \\
\hline $\begin{array}{c}\text { Zona Donostia-San Sebastián } \\
\text { (País Vasco) } \\
\begin{array}{c}\text { Zona Nantes } \\
\text { (Bretaña) } \\
\text { Total }\end{array}\end{array}$ & 5 & 452 & 637 \\
\hline
\end{tabular}

El alumnado tiene 12 años (25.1\%), 13 años (25.1\%), 14 años (24.7\%) y 15 años (25.1\%). Son chicos $(51.8 \%)$ y chicas $(49.2 \%)$.

\section{Instrumento}

Como se ha comentado anteriormente, se elaboró un cuestionario para recoger información sobre las variables objeto de estudio. Las principales dimensiones del instrumento se clasificaron en las siguientes áreas:

-Datos generales (8 ítems): Edad, sexo, nivel de satisfacción del estudiante, utilización del ordenador para escribir textos, consumo de videojuegos, Internet y televisión y situación académica.

- Actividades de aprendizaje: Escala de likert de 1 a 4 formada inicialmente por un total de 40 ítems.

Tabla 2

Dimensiones del cuestionario

\begin{tabular}{lcc}
\hline \multicolumn{1}{c}{ Dimensiones } & № ítem & Alfa Cronbach \\
\hline Aprendizaje por descubrimiento- indagación & $23,24,26,34,35,36$ y 39 & .701 \\
Ámbito afectivo-emocional & $37,40,41,42,43$ y 46 & .689 \\
Aprendizaje funcional & $9,11,15,16$ y 47 & .527 \\
Autorregulación del aprendizaje & $22,44,45$ y 48 & .611 \\
Gestión de la información & $12,27,28$ y 31 & .653 \\
Habilidades instrumentales & 29 y 30 & .506 \\
\hline
\end{tabular}

Para diseñar el cuestionario, además de revisar la literatura relacionada con el tema (Alonso, Gallego \& Honey, 2012; Biggs, 1993; Gimeno \& Gallego, 2007; Martínez, 2007), se realizaron 4 entrevistas exploratorias (2 en el País Vasco y otras 2 en Bretaña) a los docentes de los institutos de ambas regiones. Los docentes bretones modificaron algunos datos generales y añadieron 8 ítems muy específicos relacionados con sus propios 
centros educativos (deberes, actividades extraescolares, tiempo libre, motivación del estudiante, normas de convivencia, comedor del centro, lectura y lengua bretona) y que no constan en este artículo por no ser comunes a ambas regiones. El instrumento también fue validado por dos expertos de la Universidad de Zaragoza y otros tantos de la Universidad del País Vasco.

Se llevó a cabo una prueba piloto con 13 estudiantes de las dos regiones (6 de Bretaña y 7 del País Vasco) participantes en este estudio. Se seleccionaron a éstos en función de su edad (entre 12 y 15 años) y sexo (6 chicos y 7 chicas). Se modificó la redacción de 12 ítems y se clarificaron las instrucciones de la prueba, fundamentalmente por cuestiones idiomáticas. El cuestionario final contiene 48 ítems. En ellos se analizan las características del alumnado (ítems dicotómicos) y el grado de acuerdo en una escala Likert (1: Poco/Nada de acuerdo; 4: Mucho/Totalmente de acuerdo) en relación con las diferentes actividades de aprendizaje utilizadas en clase.

El análisis de la fiabilidad y validez supuso la eliminación de 12 elementos, quedando finalmente una escala de 28 ítems validada mediante análisis factorial exploratorio en 6 dimensiones $(\mathrm{KMO}=.795$; Varianza $=48.69 \%)$ y con un Alfa de Cronbach total de .803 .

\section{Procedimiento de recogida y análisis de datos}

El diseño de este estudio es cuantitativo, del tipo ex post facto y basado en la recogida de datos objetivos. Como se ha mencionado anteriormente, se pretende analizar las opiniones del alumnado sobre las actividades de enseñanza y aprendizaje basadas en la indagación mediante un cuestionario. El contacto inicial con los centros de Bretaña se realizó mediante la mediación de uno de los institutos de la zona de San Sebastián participante en este estudio y, posteriormente, por los miembros del equipo de investigación. La recogida de información se realizó entre los meses de enero y junio de 2015, una vez conseguido el consentimiento informado de las familias. La aplicación de las pruebas ha sido realizada por un coordinador o coordinadora de los docentes de los centros educativos seleccionados, siguiendo la asesoría de uno de los miembros del equipo de investigación designado a tal efecto.

Los datos de la parte cuantitativa fueron tratados con el paquete informático SPSS 22.0 e ITEMAN, realizándose diversos análisis estadísticos. Los resultados descriptivos se muestran mediante estadísticos comunes como la puntuación promedio y la desviación típica para las variables numéricas y porcentajes para las variables categóricas. Para calcular las diferencias de medias entre los estudiantes se ha utilizado la prueba U de Mann-Whitney, debido al incumplimiento de la hipótesis de normalidad, en todo caso se ha trabajado con un nivel de significación del .05. Para validar la escala se utilizó el análisis factorial y la fiabilidad como consistencia interna se estudió mediante el alfa de Cronbach.

\section{Resultados}

A continuación se van a exponer algunos resultados del estudio realizado en las dos regiones. Respecto al primer objetivo, los estudiantes afirman ir a gusto a clase (59.9\%), juegan con los videojuegos más de dos horas diarias (19.6\%), utilizan Internet 
más de dos horas diarias (5.8\%), ven la televisión más de dos horas diarias (23.5\%) $\mathrm{y}$, en el momento de la administración del cuestionario, tienen aprobadas todas las asignaturas (72.2\%).

En la Tabla 3 se identifican las actividades de aprendizaje de más interés para el alumnado de la muestra. En este caso, se analizan los promedios de las actividades de aprendizaje basadas en la indagación, la gestión de la información y el aprendizaje funcional.

Tabla 3

Actividades de aprendizaje de más interés para los estudiantes de ambas regiones I

\begin{tabular}{|c|c|c|}
\hline Cómo me gusta aprender en clase & $\mathrm{X}$ & DT \\
\hline \multicolumn{3}{|l|}{ Ámbito: Aprendizaje por descubrimiento-indagación } \\
\hline 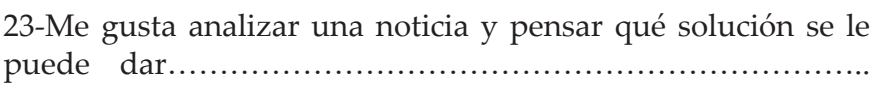 & 2.74 & .878 \\
\hline 24-Me gusta analizar por qué suceden las cosas.................. & 3.02 & .885 \\
\hline 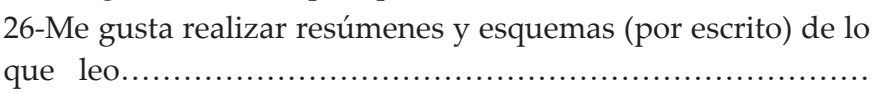 & 2.13 & .989 \\
\hline 34-Me gusta debatir en clase sobre un tema...................... & 2.81 & 1.003 \\
\hline 35-Me gusta que me pidan mi opinión en clase................... & 2.71 & .971 \\
\hline 36-Me gusta que me pregunte el profesor/a...................... & 2.36 & .940 \\
\hline 39-Me gusta presentar trabajos oralmente ....................... & 2.56 & 1.066 \\
\hline \multicolumn{3}{|l|}{ Ámbito: Aprendizaje funcional } \\
\hline $\begin{array}{l}\text { 9-Me gusta moverme en clase mientras trabajo, tener libertad } \\
\text { de movimientos en clase } \ldots \ldots \ldots \ldots \ldots \ldots \ldots \ldots \ldots \ldots \ldots \ldots \ldots \ldots \ldots \ldots \ldots \ldots \ldots \ldots \ldots \ldots\end{array}$ & 2.90 & .937 \\
\hline $\begin{array}{l}\text { 11-Me gusta hablar con mis compañeros/as mientras trabajo, } \\
\text { comunicarme con ellos.................................... }\end{array}$ & 3.02 & .959 \\
\hline 15-Me gusta conocer problemas que suceden en la calle......... & 3.17 & .816 \\
\hline 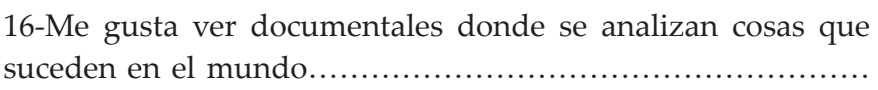 & 2.91 & .952 \\
\hline 17-Me gusta trabajar más en equipo que solo....................... & 3.14 & .918 \\
\hline Ámbito: Gestión de la información & & \\
\hline $\begin{array}{l}\text { 12-Me gusta utilizar máquinas } y \text { aparatos tecnológicos } \\
\text { (ordenador, aparatos...) para aprender........................ }\end{array}$ & 2.95 & .994 \\
\hline 27-Me gusta clasificar mis archivos de texto en el ordenador... & 2.58 & 1.045 \\
\hline 28-Me gusta buscar información en internet.................... & 3.07 & .844 \\
\hline 31-Me gusta escribir textos en el ordenador...................... & 2.63 & 1.005 \\
\hline
\end{tabular}

En la tabla anterior, se puede observar que, en relación el ámbito del aprendizaje por descubrimiento e indagación, existe una actividad que supera los tres puntos. Se trata del ítem Me gusta analizar por qué suceden las cosas. En el ámbito del aprendizaje funcional, las actividades Me gusta hablar con mis compañeros/as mientras trabajo, comunicarme con ellos, 
Me gusta conocer problemas que suceden en la calle y Me gusta trabajar más en equipo que solo son las que superan esta puntuación. Finalmente, respecto al ámbito de la gestión de la información, los estudiantes afirman que les gusta buscar información en internet.

En la Tabla 4 se analizan los promedios de las actividades de aprendizaje relacionadas con la auto-regulación y el ámbito afectivo-emocional.

Tabla 4

Actividades de aprendizaje de más interés para los estudiantes de ambas regiones II

\begin{tabular}{|c|c|c|}
\hline Cómo me gusta aprender en clase & $\mathrm{x}$ & DT \\
\hline \multicolumn{3}{|l|}{ Ámbito: Afectivo y emocional } \\
\hline 37-Me gusta que me escuche el profesor/a.. & 3.14 & .884 \\
\hline 40-Me gusta sacar muy buenas notas en clase.................. & 3.70 & .647 \\
\hline 41-Me gustaría ser de los mejores alumnos/as de la clase...... & 3.05 & .926 \\
\hline 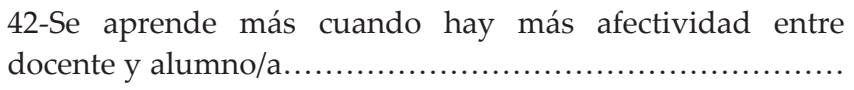 & 3.07 & .884 \\
\hline 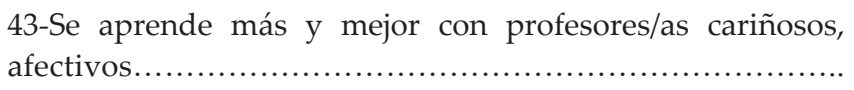 & 3.02 & .930 \\
\hline 46-Me gusta que me feliciten cuando hago bien las cosas...... & 3.23 & .875 \\
\hline \multicolumn{3}{|l|}{ Ámbito: Autorregulación del aprendizaje } \\
\hline 22-Cuanto más sabes en el colegio más sabes en la vida....... & 2.93 & .928 \\
\hline 44-Me gusta repasar las cosas (autorregulación)................ & 2.54 & .955 \\
\hline 45-Me gusta autoevaluarme y no que me evalúen los demás. & 2.56 & 1.043 \\
\hline 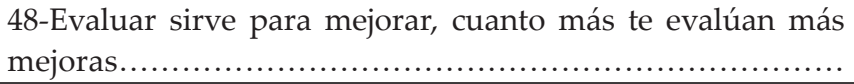 & 2.78 & .973 \\
\hline
\end{tabular}

Llama poderosamente la atención que todas las actividades de aprendizaje del ámbito afectivo y emocional superan los tres puntos. Los siguientes ítems son muy valorados por los estudiantes de la muestra: Me gusta sacar muy buenas notas en clase, Me gusta que me feliciten cuando hago bien las cosas, Me gusta que me escuche el profesor/a, Se aprende más cuando hay más afectividad entre docente y alumno/a, Me gustaría ser de los mejores alumnos/as de la clase y Se aprende más y mejor con profesores/as cariñosos, afectivos. Los estudiantes de ambas regiones otorgan mucha importancia a ambas facetas del aprendizaje. Respecto al ámbito de la auto-regulación del aprendizaje, no hay ninguna actividad que supere esta puntuación. El ítem mejor puntuado se refiere al valor educativo de la escuela para afrontar la vida (Cuanto más sabes en el colegio más sabes en la vida).

La identificación y comparación de las puntuaciones obtenidas por los estudiantes con y sin problemas académicos en relación con las actividades de aprendizaje desarrolladas en el aula pueden aportar algunos datos interesantes sobre las preferencias de ambos colectivos.

En la Tabla 5, se exponen las diferencias de medias entre las opiniones de estos dos grupos de estudiantes, en función de su trayectoria académica (ítem 7 del cuestionario). 


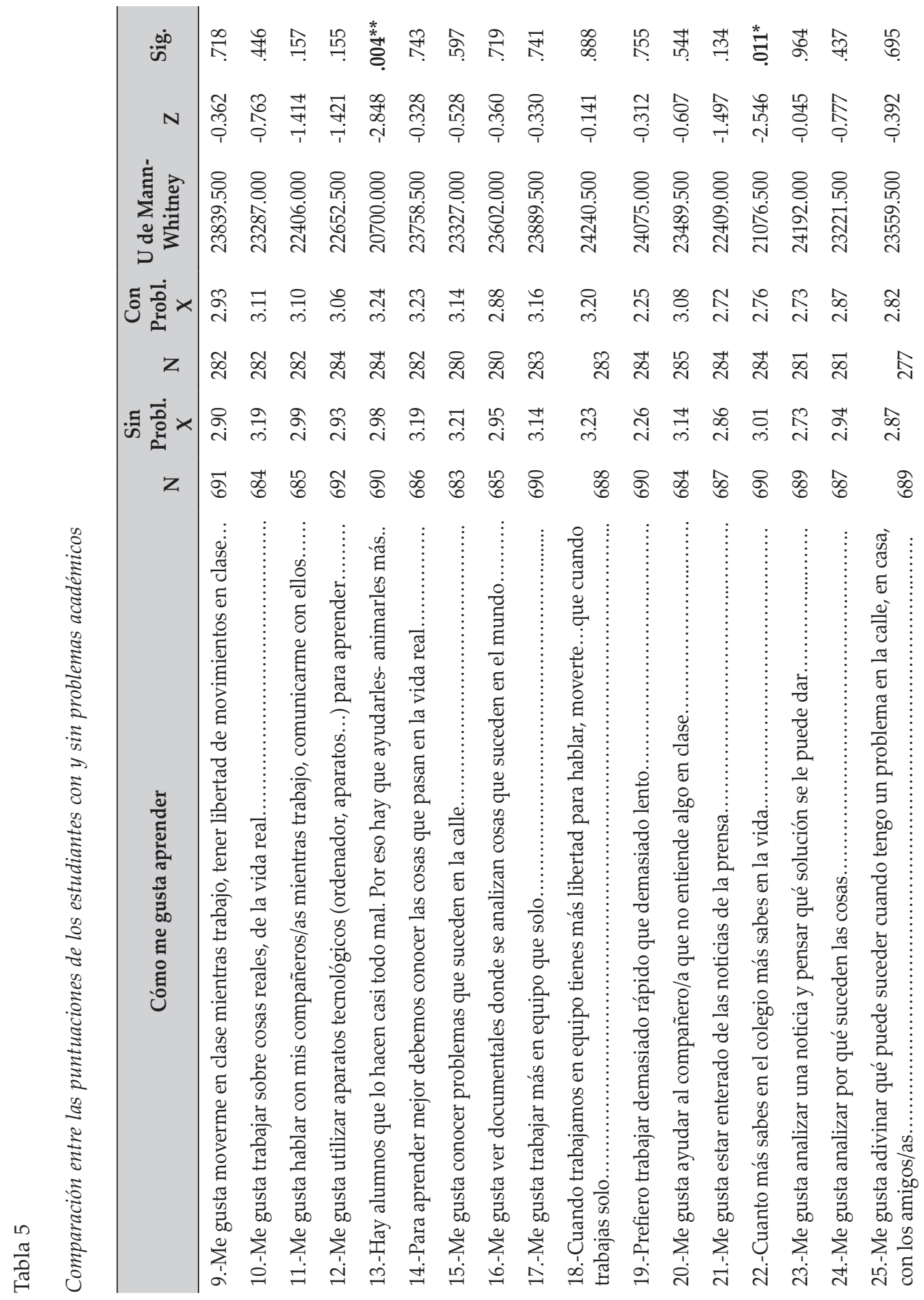




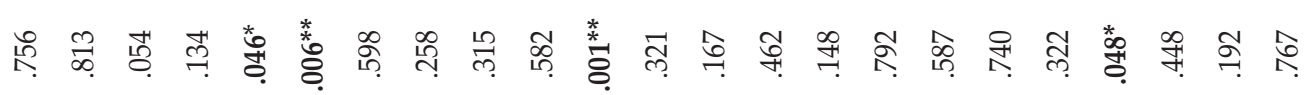

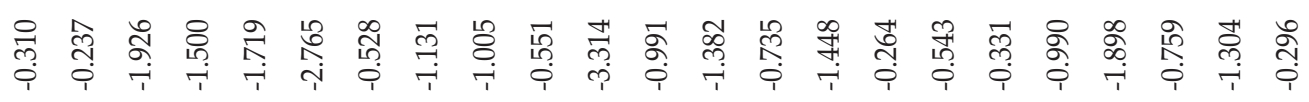

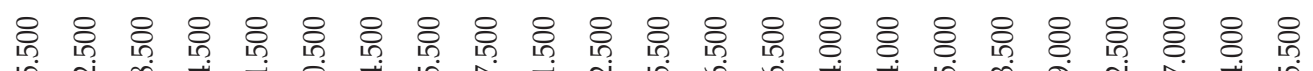

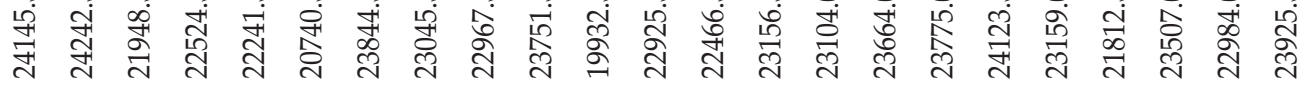

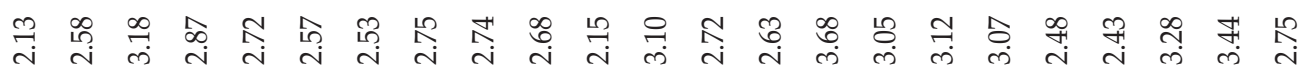

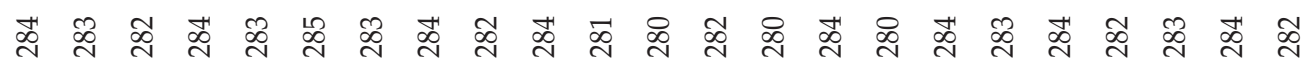

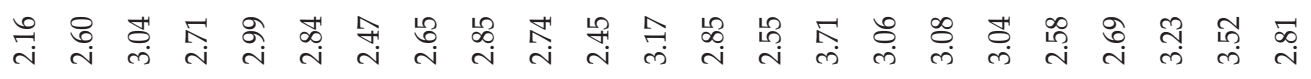

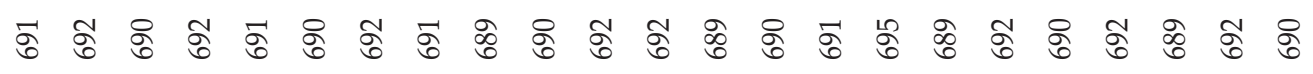

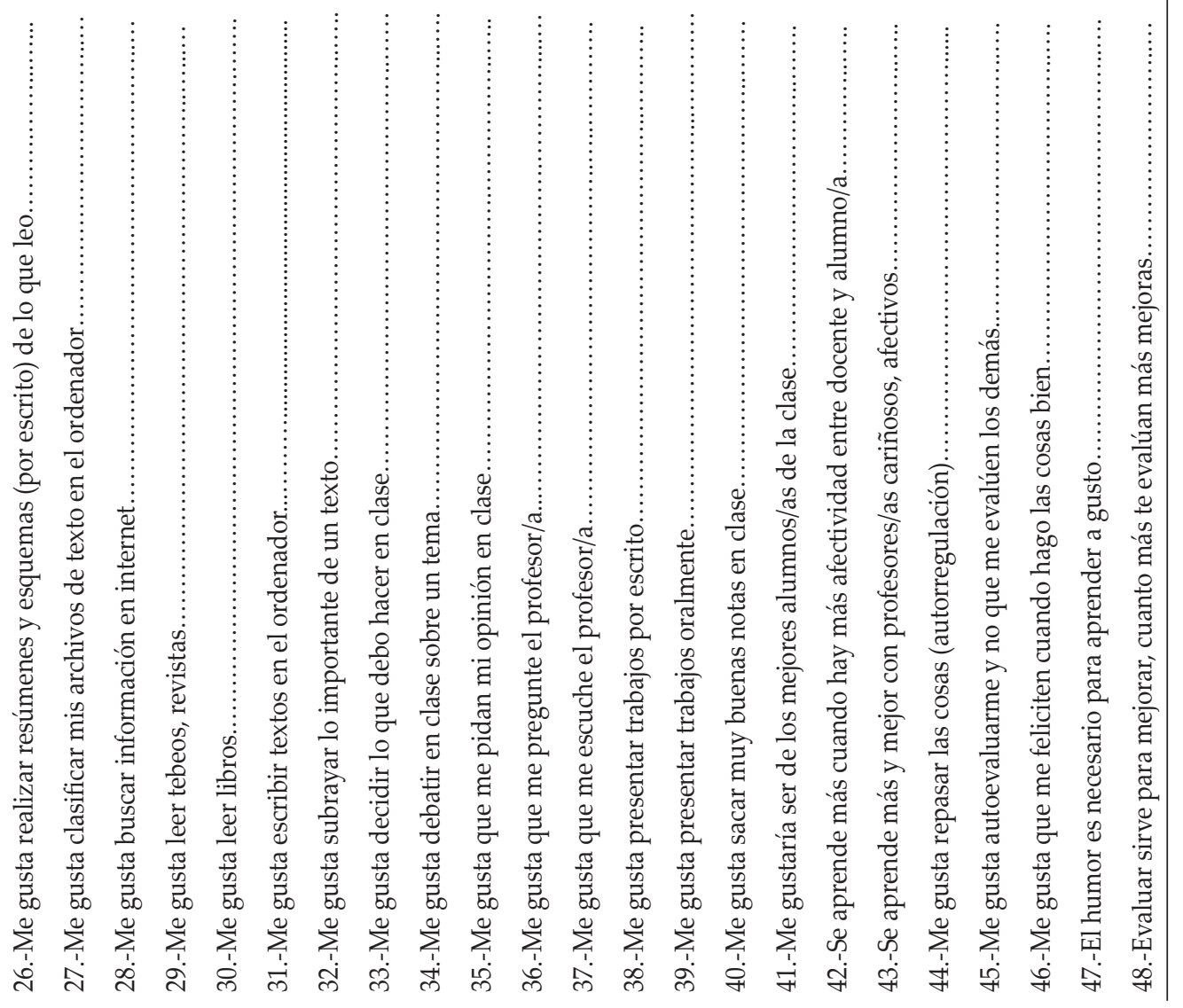


Al realizar la comparación de medias entre el alumnado con y sin problemas académicos, se puede observar que existen seis ítems con diferencias significativas: la ayuda dirigida al alumnado con dificultades, la confianza en la labor educativa de la escuela, el gusto por la lectura y la escritura, por la autoevaluación del aprendizaje y la participación en clase (que le pregunte el profesor/a).

\section{Discusión y Conclusiones}

El análisis de los resultados de las opiniones del alumnado de las zonas de San Sebastián y Nantes nos lleva a extraer las siguientes conclusiones:

Respecto al primer objetivo del estudio (Analizar las características del alumnado de educación secundaria obligatoria participante en el estudio), los estudiantes consultados tienen una edad entre doce y quince años, afirman ir a gusto a clase, juegan con los videojuegos menos de dos horas diarias, utilizan frecuentemente internet, ven poco la televisión y, la mayoría, hasta ahora, tienen aprobadas todas las asignaturas.

En relación con el segundo objetivo (Describir las opiniones de los estudiantes de las dos regiones -Bretaña y País Vasco- sobre las estrategias de enseñanza y aprendizaje basadas en la indagación). En los resultados del estudio se constata la importancia concedida por el alumnado al ámbito emocional y a las actividades relacionadas con la indagación y el descubrimiento, el conocimiento de problemas, el trabajo en equipo (comunicarse...) y la búsqueda de información (internet). Estas afirmaciones van en la línea del estudio de Chen y Howard (2010) cuando constatan que la actitud hacia el aprendizaje del alumnado de educación secundaria mejora cuando se fomentan la búsqueda y la gestión de la información y se desarrollan procesos de indagación vinculados a la vida cotidiana.

Respecto al tercer objetivo (Analizar y comparar las puntuaciones obtenidas por los estudiantes con y sin problemas académicos en relación con las actividades de aprendizaje desarrolladas en el aula), se puede concluir que los y las estudiantes que han superado todas las asignaturas confían en la labor educativa de la escuela, les gusta participar en clase (que le pregunte el profesor/a), autoevaluar su trabajo y trabajar las técnicas instrumentales (lectura y escritura). En este sentido, Escribano, Bejarano, Zúñiga y Fernández (2010) afirman que el acto de aprendizaje puede frustrarse cuando el estudiante no ha llegado a un grado de dominio de habilidades básicas suficiente (comprensión lectora, expresión escrita...) en proporción a las exigencias de ese aprendizaje. Los estudiantes precisan de una base sólida y previa que sirva de andamio para desarrollar futuros aprendizajes basados en la indagación abierta y en la cooperación entre compañeros y compañeras. Los autores se muestran favorables a aplicar metodologías de alto control externo (instrucción directa, fundamentalmente para dominar los conocimientos básicos) con el alumnado que tiene dificultades, para posteriormente, después de una práctica consolidada, seguir trabajando con metodologías más abiertas como, por ejemplo, el aprendizaje basado en proyectos y problemas.

En este sentido, Fernández, Rodríguez y Martínez (2015) y Vázquez y Manassero (2015) creen que una propuesta innovadora para la enseñanza secundaria pasa por el fomento de enfoques socio-constructivistas, el diseño de secuencias de enseñanza y aprendizaje orientadas a la indagación y centradas en el estudiante, la organización del aprendizaje basada en situaciones reales, el fomento de la evaluación formativa y el impulso de los 
aspectos emocionales, la auto-regulación y la meta-cognición. Para ello, es imprescindible desarrollar un currículum más interdisciplinar, reducir el número de docentes que trabajan con los estudiantes, desarrollar una educación más personalizada e impulsar las relaciones entre el profesorado y el alumnado. Es una cultura organizativa y curricular que no siempre promueven los centros educativos de enseñanza secundaria.

El alumnado consultado en el estudio, independientemente de su rendimiento académico, otorga valoraciones altas a las actividades funcionales y basadas en la indagación. Como afirman Kissling (2014) y Méndez (2015), los docentes que fomentan la transferencia de conocimientos, acercando los problemas de la vida cotidiana al aula, obtienen más fácilmente la implicación de los estudiantes. Es lo que Pineda (2015), denomina aprendizaje de alto nivel en la enseñanza secundaria. Es decir, el impuso de actividades de aprendizaje desarrollados en contextos cercanos, aprovechando situaciones reales, generando alternativas para solucionar problemas en equipo, comunicando lo aprendido y fomentando la transferencia de conocimientos a otras situaciones vitales. En este sentido, Nortes y Nortes (2016) afirman que, en la actualidad, los docentes españoles siguen fomentando procesos de indagación demasiado estructurados (el profesorado decide tanto el tipo de problema a trabajar como el procedimiento a seguir), sin llegar a impulsar modelos de indagación abierta (el problema y la resolución parten de los estudiantes). Autores como Renzulli (2010) y Lupión y Prieto (2014) concluyen que la buena educación debe desarrollar un currículum que conceda al alumnado oportunidades para desarrollar su propio estilo de aprendizaje.

Para que el aprendizaje basado en la indagación se convierta en una alternativa a la enseñanza por recepción, es necesario capacitar a los docentes. Benarroch y Marín (2011) y Sáez y Ruiz (2012) creen que la conducta docente está orientada y, a la vez, condicionada por un sistema personal e implícito de creencias. Analizan sus concepciones sobre la educación y concluyen que la formación del profesorado de secundaria tiene escasa incidencia en la transformación de las creencias pedagógicas de los docentes. Cassullo y García (2015) afirman que existe un claro déficit que conviene subsanar en los programas formativos dirigidos al profesorado de educación secundaria: el escaso impulso de la dimensión afectiva, actitudinal y moral. En este sentido, Engelbrecht, Savolainen y Nel (2013) constatan que el sistema educativo finlandés mantiene un histórico compromiso con la formación del profesorado de educación secundaria. Los educadores y educadoras deben fomentar el desarrollo socio-emocional de los estudiantes y, por ello, las instituciones encargadas de la capacitación docente deben trabajar sistemáticamente estas competencias con los futuros docentes.

La educación secundaria necesita profesionales que generen confianza y cercanía con los estudiantes (Méndez, 2015). Los buenos docentes no solamente se caracterizan por el dominio de la asignatura, sino también por su implicación en la formación de ciudadanos y ciudadanas comprometidas y responsables. Como afirman Fernández, Contini, Ongarato, Saavedra y Iglesia (2009) y Serrano y Pontes (2015), caminar hacia una educación de calidad, donde todo el alumnado desarrolla al máximo sus posibilidades, nos lleva inexorablemente a replantear el rol del docente y del estudiante en el fomento de la indagación y en el desarrollo de competencias sociales y emocionales.

Finalmente, se debe hacer mención a las limitaciones y fortalezas de la investigación. El desarrollo del estudio tuvo algunos problemas en su ejecución. Por una parte, 
la sintonía entre los coordinadores y coordinadoras de ambas regiones no fue todo lo buena que se esperaba. Y por otra, hubo bastantes dificultades con la obtención del consentimiento informado de los estudiantes de ambas regiones. En cuanto a las fortalezas, se debe subrayar la satisfacción que produce contribuir a impulsar la cooperación entre los profesionales de los centros participantes. Este estudio puede ser el inicio de futuros aprendizajes.

\section{Referencias}

Abel, M. (2012). Desde la literatura, ópera, cine y televisión hasta las ciencias jurídicas y el derecho penal mediante la nueva técnica pedagógica del aprendizaje basado en problemas. Revista Jurídica de Investigación e Innovación Educativa, 5, 87-104.

Alonso, C., Gallego, D., \& Honey, P. (2012). Los estilos de aprendizaje: procedimientos de diagnóstico y mejora. Madrid: TEA Ediciones.

Andriessen, I., Phalet. K., \& Lens, W. (2006). Future goal setting, task motivation and learning of minority and non-minority students in Dutch schools. British Journal of Educational Psichology, 76, 827-850.

Benarroch, A., \& Marín, N. (2011). Relaciones entre creencias sobre enseñanza, aprendizaje y conocimientos de ciencias. Enseñanza de las Ciencias, 29(2), 289-304. doi: http:// dx.doi.org/10.5565/rev/ec/v29n2.84

Bermúdez, G., Díaz, S., Lía, A., \& Gavidia, V. (2014). La transposición del concepto de diversidad biológica. Un estudio sobre los libros de texto de la educación secundaria obligatoria española. Enseñanza de las Ciencias, 32(3), 285-302. doi: http://dx.doi. org/10.5565/rev/ensciencias.1129

Biggs, J. (1993). What do Inventories of student's Learning processes really measure? A Theoretichal view and clarification. British Journal of Educational Psychology, 63, 3-19.

Cassullo, G. L., \& García, L. (2015). Estudio de las competencias socio emocionales y su relación con el afrontamiento en futuros profesores de Nivel Medio. Revista Electrónica Interuniversitaria de Formación del Profesorado, 18(1), 213-228. doi: ttp://dx.doi. org/10.6018/reifop.18.1.193041.

Criado, A. M., \& Guzman, M. C. (2014). ¿Cómo mejorar la educación científica de primaria en España desde el currículo oficial? Sugerencias a partir de un análisis curricular comparativo en torno a las finalidades y contenidos de la ciencia escolar. Enseñanza de las Ciencias, 32(3), 249-266. doi: http://dx.doi.org/10.5565/rev/ensciencias.1069

Chen, C.H., \& Howard, B. (2010). Effect of live simulation on middle school students attitudes and learning toward science. Educational Technology E Society, 13(1), 133-139.

Di Giusto, C., Martin, M. A., \& Arnaiz, A. (2014). Competencias personales y sociales en adolescentes. Revista Iberoamericana de Educación, 66, 89-104.

Dignath, C., \& Buettner, G. (2008). Components of fostering self-regulated learning among students. A meta-analysis on intervention studies at primary and secondary school level. Metacognition and Learning, 3(3), 231-264.

Doménech, F. (2012). Análisis de los estilos de pensamiento que utilizan los profesores españoles en el aula. Revista de Educación, 358, 497-522. doi: 10-4438/1988-592X-RE2010-358-088 
Engelbrecht, P. Savolainen, H., \& Nel, M. (2013). How cultural histories shape South African and Finnish teachers' attitudes towards inclusive education: a comparative analysis. European Journal of Special Needs Education, 28(3), 305-318.

Escribano, A., Bejarano, M. T., Zúñiga, M. A., \& Fernández, J. L. (2010). Programa de metodología didáctica para la mejora de la inteligencia emocional y el Aprendizaje Basado en Problemas (ABP). Revista Docencia e Investigación, 20, 271-305.

Fernández, M., Contini, N., Ongarato, P., Saavedra, E., \& Iglesia, G. (2009). Estrategias de afrontamiento frente a problemas académicos en estudiantes medios y universitarios. Revista Iberoamericana de Diagnóstico y Evaluación, 27(1), 63-84.

Fernández, M. J., Rodríguez, J. M., \& Martínez, A. (2015). Práctica docente del profesorado de educación secundaria obligatoria en España según TALIS 2013. Revista Española de Pedagogía, 261, 219-224.

Gasco, J. (2016). El empleo de estrategias en el aprendizaje de las Matemáticas en la Enseñanza Secundaria Obligatoria. Revista de Investigación Educativa, 34(2), 487-502. doi: http://dx.doi.org/10.6018/rie.34.2.222901

Gillies, R. (2008). The effects of cooperative learning on junior high school students bahaviours, discourse and learning during a science-based learning activity. School Psychology International, 29(3), 328-347.

Gimeno, M., \& Gallego, S. (2007). La autoevaluación de las competencias básicas del estudiante de Psicología. Revista de Psicodidáctica, 12(1), 7-28.

Hinojosa, J., \& Sanmartí, N. (2015). La autorregulación metacognitiva como medio para facilitar la transferencia en mecánica. Revista Eureka sobre enseñanza y divulgación de las ciencias, 12(2), 249-263

Kissling, M. T. (2014). Now and then, in and out of the classroom: Teachers learning to teach through the experiences of their living curricula. Teaching and Teacher Education, 44, 81-91.

La Vanguardia (29 de Noviembre de 2002). Francia saca a las escuelas bretonas del sistema de educación público. La Vanguardia. Recuperado de http://www.lavanguardia. com/cultura/20021129/51262765427/francia-saca-a-las-escuelas-bretonas-del-sistemade-educacion-publico.html

Lara, L. F., \& Samper, C. (2015). Logros y desaciertos cuando se aprende a demostrar. Enseñanza de las Ciencias, 33(2), 113-132. doi: http://dx.doi.org/10.5565/rev/ensciencias.1491

Lavonen, J., \& Laaksonen, S. (2009). Context of Teaching and Learning School Science in Finland: Reflections on PISA 2006 Results. Journal of Research in Science Teaching, 46(8), 922-944.

Lupión, T., \& Prieto, T. (2014). La contaminación atmosférica: un contexto para el desarrollo de competencias en el aula de secundaria. Enseñanza de las Ciencias, 32(1), 159-177. doi: http://dx.doi.org/10.5565/rev/ensciencias.830

Martínez, P. (2007). Aprender y enseñar. Estilos de aprendizaje y de enseñanza desde la práctica de aula. Bilbao: Mensajero.

Martínez, A. Piqueras, J.A., \& Inglés, C. J. (2011). Relaciones entre la inteligencia emocional y estrategias de afrontamiento ante el estrés. Revista Electrónica de Motivación y Emoción, 14, 1-24.

Méndez, D. (2015). Estudio de las motivaciones de los estudiantes de secundaria de física y química y la influencia de las metodologías de enseñanza en su interés. Educación XX1, 18(2), 215-235. doi: 10.5944/educXX1.14016 
Mengascini, A., \& Mordeglia, C. (2014). Caracterización de las prácticas experimentales en la escuela a partir del discurso de docentes de primaria y secundaria. Enseñanza de las Ciencias, 32(2), 71-89. doi: http://dx.doi.org/10.5565/rev/ensciencias.755

Nortes, R., \& Nortes, A. (2016). Resolución de problemas, errores y dificultades en el grado de maestro de primaria. Revista de Investigación Educativa, 34(1), 103-117. doi: http://dx.doi.org/10.6018/34.1.229501

Pineda, J. A. (2015). Educar para la ciudadanía trabajando con temas controvertidos en Educación Secundaria Obligatoria. Revista de Investigación Educativa, 33(2), 353-367. doi: http://dx.doi.org/10.6018/rie.33.2.208441

Prieto, A., Barbarroja, J., Reyes, E., Monserrat, J., Díaz, D., Villarroel, M., \& Álvarez, M. (2006). Un nuevo modelo de aprendizaje basado en problemas, el ABP 4x4, es eficaz para desarrollar competencias profesionales valiosas en asignaturas con más de 100 alumnos. Aula Abierta, 87, 171-194.

Recio, M. A. (2014). El aprendizaje basado en la resolución de problemas: una experiencia práctica. Docencia y Derecho. Revista para la docencia jurídica universitaria, 7, 1-8.

Renzulli, J. (2010). El rol del profesor en el desarrollo del talento. REIFOP, 13(1). Recuperado de http://m.aufop.com/aufop/uploaded_files/articulos/1268615182.pdf

Reschly, A. L., Huebner, E.S., Appleton, J. J., \& Antaramian, S. (2008). Engagement as flourishing. The contribution of positive emotions and coping to adolescents engagement at school and with learning. Psychology in the Schools, 45(5), 419-431.

Sáez, J. M., \& Ruiz, J. M. (2012). Estrategias metodológicas, aprendizaje colaborativo y TIC: un caso en la Escuela Complutense Latinoamericana. Revista Complutense de Educación, 23(1), 115-134.

Serrano, R.,\& Pontes, A. (2015). Expectativas ante la formación inicial entre el alumnado del Máster de Profesorado de Enseñanza Secundaria. Revista de Investigación Educativa, 33(2), 489-505. doi: http://dx.doi.org/10.6018/rie.33.2.203471

Sideridis, G.D., \& Kaplan, A. (2011). Achievement Goals and Persistence Across Tasks: The Roles of Failure and Success. The Journal of Experimental Education, 79(4), 429-451.

Studsrod, I., \& Bru, E. (2011). Perceptions of peers as socialization agents and adjustment in upper secondary school. Journal: Emotional and Behavioural Difficulties, 16(2), 159-172.

Vázquez, A., \& Massanero, M. A. (2015). Hacia una formación inicial del profesorado de ciencias basada en la investigación. Revista Española de Pedagogía, 261, 343-363.

Vilchez, J. M., \& Bravo, B. (2015). Percepción del profesorado de ciencias de educación primaria en formación acerca de las etapas y acciones necesarias para realizar una indagación escolar. Enseñanza de las Ciencias, 33(1), 185-202. doi: http://dx.doi. org/10.5565/rev/ensciencias.1529

Zamora, A., \& Ardura, D. (2014). ¿En qué medida utilizan los estudiantes de Física de Bachillerato sus propios errores para aprender? Una experiencia de autorregulación en el aula de secundaria. Enseñanza de las Ciencias, 32(2), 253-268. doi: http://dx.doi. org/10.5565/rev/ensciencias.1067

Fecha de recepción: 30 de diciembre de 2016.

Fecha de revisión: 26 de mayo de 2017.

Fecha de aceptación: 26 de mayo de 2017. 